\title{
Elevated expression of c-fos in central nervous system correlates with visceral hypersensitivity in irritable bowel syndrome (IBS): a new target for IBS treatment
}

\author{
Ru Zhang • Ning Zou • Ji Li • Hong Lv • Jing Wei • \\ Xiu-Cai Fang • Jia-Ming Qian
}

Accepted: 1 February 2011 / Published online: 22 February 2011

(C) The Author(s) 2011. This article is published with open access at Springerlink.com

\begin{abstract}
Background and aims Although visceral hypersensitivity is a major pathophysiological feature of irritable bowel syndrome (IBS), its molecular mechanisms are still poorly understood. c-fos is a well-established marker of cell activation. Accumulating evidence demonstrates that norepinephrine (NE) system is dysregulated in IBS; however, very little is known on its mechanism. It is our hypothesis that elevated expression of $c$-fos in central nervous system (CNS) correlates with visceral hypersensitivity in rat model of IBS. Furthermore, we explored the changes of NE system in IBS patients.

Methods The rat model of IBS was induced by heterotypic chronic and acute stress. Tissues obtained from rat model were analyzed for c-fos levels in CNS (frontal lobe, hippocampus, cornu dorsale) and colon by immunohistochemistry. Real-time reverse transcription polymerase chain reaction was used to detect tyrosine hydroxylase $(\mathrm{TH})$ in the colonic tissues obtained from IBS patients.
\end{abstract}

\footnotetext{
R. Zhang $\cdot$ N. Zou $\cdot$ J. Li $\cdot$ H. Lv $\cdot$ X.-C. Fang $\cdot$ J.-M. Qian $(\bowtie)$ Department of Gastroenterology,

Peking Union Medical College Hospital,

Peking Union Medical College,

Chinese Academy of Medical Sciences,

1 Shuaifuyuan Wangfujing Street,

Beijing 100730, China

e-mail: qjiaming57@gmail.com

J. Wei

Department of Psychologic Medicine,

Peking Union Medical College Hospital,

Peking Union Medical College,

Chinese Academy of Medical Sciences,

1 Shuaifuyuan Wangfujing Street,

Beijing 100730, China
}

Results The rat model of IBS was associated with increased expression of c-fos in different parts of CNS ( $P=0.001, P=$ 0.002 , and $P=0.002$, respectively), but normal in colon $(P=$ 0.207). The clinical parameters (colonic motility and sensation) of rat model were significantly correlated with elevated c-fos in CNS $(P<0.05)$. Enterochromaffin cells and serotonin in colon were related to the elevated $\mathrm{c}-$ fos in CNS $(P<0.05)$. The TH messenger ribonucleic acid (mRNA level of IBS-D patients was almost four times as much as that of controls.

Conclusions Elevated expression of c-fos in CNS might be one of key mechanisms in etiology of IBS. Therefore, regulation of CNS activation could be a major targeting effect when treating IBS patients.

Keywords Irritable bowel syndrome $\cdot \mathrm{c}$-fos .

Norepinephrine $\cdot$ Tyrosine hydroxylase

\section{Introduction}

Irritable bowel syndrome (IBS) is a highly prevalent functional disorder of the gastrointestinal (GI) tract characterized by the presence of abdominal pain or discomfort, an alteration in bowel habit, and the absence of reproducible biomarkers [1]. Several pathophysiological mechanisms have been reported to play a role in the pathogenesis of IBS, among which are visceral hypersensitivity [2], central nervous system (CNS) dysregulation [3], alterations of gastrointestinal (GI) motility [4], and abnormalities in neurotransmitter systems such as serotonin (5-HT) [5]. However, the definite mechanism is still poorly understood. There is general agreement that increased sensitivity to stimuli arising from the gut wall (i.e., visceral hypersensitivity) contributes to abdominal pain/discomfort in IBS patients [6]. 
The causes underlying the visceral hypersensitivity in IBS are only partially understood. Recent studies on the pathophysiology of visceral hypersensitivity have demonstrated abnormal neuroendocrine reactions within the gut wall. There is evidence for altered signaling at the mucosal level as indicated by studies using mucosal biopsy supernatants from IBS patients. Soluble factors released from colonic mucosal specimens of IBS patients activate visceral afferent neurons and induce visceral hypersensitivity in rats [7] and mice [8]. After measuring serotonin, histamine and tryptase concentrations in colonic biopsy specimens from patients with IBS and controls, Buhner et al. [9] concluded that altered signaling in mucosa and the enteric nervous system might be involved in IBS pathogenesis.

Norepinephrine (NE) is one of the most important neurotransmitters within the GI tract $[10,11]$. The generation of $\mathrm{NE}$ is rate limited by tyrosine hydroxylase (TH) [12]. TH is the first rate-limiting enzyme in the process of NE synthesis, whose activity affects synthetic process of NE. Lately, most attention has been focused on visceral hypersensitivity related to dysfunction of the $\mathrm{NE}$ system. Some authors raised the hypothesis that NE signaling might be involved in the visceral hypersensitivity of IBS. Several investigators measuring blood, urine, or saliva noted higher levels of NE in patients with IBS relative to healthy controls (HCs) [13-19]. Kim et al. [20] found association of distinct alpha (2) adrenoceptor polymorphisms with constipation and somatic symptoms in functional GI disorders, but did not find association of norepiphrine transporter (NET) with IBS. Until now, most studies put emphasis on NE signaling in body fluid (plasma, urine, saliva, cerebrospinal fluid, and so on) of IBS, and the results were liable to be influenced. But NE signaling in tissue (such as colonic mucosa) of IBS has been scarcely studied yet. Since our prior study has found that NE system (NET and $\alpha_{2 \mathrm{~A}}-\mathrm{AR}$ ) in colon of IBS model altered significantly in comparison with controls [21], we further investigated the significance of TH in IBS in the study.

c-fos expression is a well-established marker of neuronal activation, and immunohistochemical detection of c-fos allows a mapping of activated nervous tissues on a singlecell level [22]. Some studies have shown that noxious distension of hollow viscera (i.e., colorectum, esophagus, and stomach) induces a specific pattern of c-fos expression in the rat spinal cord and some of brain nuclei [23]. Little is known about the activity of CNS and GI tract in patients with IBS. Therefore, in the present study, we used the brain-gut interaction animal model of IBS which we established in our laboratory to identify activity of CNS and colon, as assessed by induction of c-fos expression.

The previous studies largely emphasized the significance of serotonin signaling in colon from patients with IBS, whereas very limited study about NE signaling at colonic mucosal level was carried out. In this study, we first investigated NE signaling in human colonic mucosa of IBS patients with two aims. First, the brain-gut interaction animal model [the combined chronic and acute stress (CAS) rats] of our lab had been validated and can simulate the disturbed sensory-motor function of gut, visceral hypersensitivity, mental behaviors of patients with IBS-D [21]. Hence, we used this model to test the hypothesis that altered c-fos, a well-established marker of cell activation, in CNS might be the probable molecular mechanism of visceral hypersensitivity in IBS. Second, this was also carried out to evaluate changes of TH in colonic mucosa of patients with IBS and to compare the expression of TH and c-fos between IBS and controls and the probable molecular mechanism.

\section{Materials and methods}

\section{Rat model of IBS}

Wistar male rats weighing 200-220 g each were used for the experimental procedures. The rats were allowed 1 week to acclimate to the surroundings before beginning the experiment. The animals were housed in individual plastic cages $(25 \times 25 \times 35 \mathrm{~cm})$. Standard rat food and tap water were available ad libitum for the duration of the experiments unless otherwise noted. The temperature was maintained at $22 \pm 2{ }^{\circ} \mathrm{C}$. A $12 / 12 \mathrm{~h} \mathrm{light/dark}$ cycle was maintained unless otherwise noted. Then the rats were randomly divided into three groups. Group 1, the control animals, were left undisturbed in the home cages with the exception of general handling. In Group 2, the chronic stress (CS group, the chronic mild stress procedure was designed to maximize the unpredictable nature of the stressors. The CS group was exposed to the following seven stressors at random order: overnight illumination for $12 \mathrm{~h}, 45^{\circ} \mathrm{C}$ hot environment for $5 \mathrm{~min}$, water deprivation for $24 \mathrm{~h}, 4^{\circ} \mathrm{C}$ cold environment for $3 \mathrm{~min}$, tail clamp for $1 \mathrm{~min}$, level vibration $(120 / \mathrm{min})$ for $40 \mathrm{~min}$, and food deprivation for $24 \mathrm{~h}$. The stressors were presented in the order shown during the 1st week and were repeated each of the weeks for a total of 3 weeks. Group 3, the CAS group [21], was treated as CS group first and, after 7 days of rest, $1 \mathrm{~h}$ of acute restraint stress was given to these group of rats. The frontal lobe, hippocampus, cornu dorsale, and distal colon were taken from rat models and stored at $-80^{\circ} \mathrm{C}$.

The rats' clinical parameters were as follows: The body weight gain can be an indicator of the rats' general state. The number of fecal pellets expelled in $1 \mathrm{~h}$ was used as an indirect measure of colonic motility [24]. Colonic sensation was represented by abdominal muscle contractions under 
different levels $(0,0.4,0.8$, and $1.2 \mathrm{ml})$ of colorectal balloon distension (CRD).

Analysis of c-fos, EC cells, and serotonin (5-HT) by histology and immunohistochemistry

Rats were anesthetized by sodium pentobarbital, and all efforts were made to minimize suffering. The frontal lobe, hippocampus, cornu dorsale, and distal colon specimens were collected. All the tissues were fixed in buffered $10 \%$ formalin. Paraffin-embedded specimens were cut with a microtome at 3-4 $\mu \mathrm{m}$, mounted on to gelatin-coated slides, and processed for either hematoxylin-eosin (HE) stain histology or immunohistochemistry. Paraffin sections were deparaffinized before processing. The antigen was retrieved by microwaving at $750 \mathrm{~W}$ for $2 \mathrm{~min}$ in $0.01 \mathrm{~mol} / \mathrm{l}$ tri-sodium citrate buffer, $\mathrm{pH}$ 6.0. $3 \%$ hydrogen peroxide for $10 \mathrm{~min}$ in order to block endogenous peroxidase activity, and sections were incubated with rabbit monoclonal antibodies directed against c-fos (diluted at 1:800; Santa Cruz, USA), chromogranin A (diluted at 1:50; Neuromaker, USA), or serotonin (diluted at 1:150; Neuromaker), respectively, overnight at $4^{\circ} \mathrm{C}$. Following thorough washing with paraformaldehyde in phosphatebuffered saline (PBS), slides were reincubated for $30 \mathrm{~min}$ at $37^{\circ} \mathrm{C}$ with secondary goat anti-mouse-rabbit antibodies (1:200; Gene Tech, USA) followed by horseradish peroxidase conjugate. 3,3'-Diamino-benzidine-tetrahydrochloride (DAB) solution prepared by dissolving one DAB-buffer tablet in $10 \mathrm{ml}$ distilled water for $3 \mathrm{~min}$. All incubation steps were performed at room temperature. Between steps, the sections were washed three times for $2 \mathrm{~min}$ with PBS $(0.01 \mathrm{~mol} /$ 1 phosphate buffer, $\mathrm{pH}$ 7.4) and with distilled water after $\mathrm{DAB}$ staining. All dilutions were in PBS, containing 0.1\% BSA (Sigma) for dilution of the primary antibody. Controls for the specificity of immunohistochemistry included a foregone positive section of human colon carcinoma is used as positive control, the replacement of the primary antibody with PBS as negative control, and the normal colonic tissue as own control.

Quantification of c-fos was performed with an Olympus $\mathrm{CH}-2$ microscope using a computer-assisted analysis system. Briefly, this method adapts a morphometric point-counting technique to quantify the area covered by c-fos in the rats' tissues. The results were expressed as opacity density (OD).

Study participants and tissue samples

Patients $[n=29 ; 19$ with diarrhea-predominant IBS (IBS-D) and 10 with constipation-predominant IBS (IBS-C); Table 1] and healthy volunteers $(n=15)$ were recruited in the Department of Gastroenterology, Peking Union Medical College Hospital and were diagnosed according to the Rome II criteria. Biopsy samples were taken when the patients were symptomatic. Generally, patients were in their flare of symptoms, and colonoscopy was performed shortly after consultation.

To evaluate the symptoms, each participant completed a validated bowel disease questionnaire (including questions that corresponded to Rome II criteria), self-rating depression scale, and self-rating anxiety scale. The symptoms surveyed were abdominal distention, mucous stool, difficult defecation, defecation urgency, incomplete defecation, and difficulty sleeping. We have grouped patients by symptoms at the time of the questionnaire into IBS-D group and IBS-C group.

Table 1 Association between c-fos in CNS and clinical parameters of IBS model

\begin{tabular}{|c|c|c|c|c|}
\hline & & $\mathrm{c}$-fos in frontal lobe & c-fos in hippocampus & c-fos in cornu dorsale \\
\hline \multirow[t]{2}{*}{ Body weight gain } & $R$ & -0.763 & -0.958 & -0.929 \\
\hline & $P$ value & 0.017 & 0.000 & 0.000 \\
\hline \multirow[t]{2}{*}{ Colonic motility } & $R$ & 0.831 & 0.656 & 0.725 \\
\hline & $P$ value & 0.005 & 0.055 & 0.027 \\
\hline \multirow[t]{2}{*}{ Visceral sensation $(0 \mathrm{ml})$} & $R$ & 0.823 & 0.776 & 0.706 \\
\hline & $P$ value & 0,006 & 0.014 & 0.034 \\
\hline \multirow[t]{2}{*}{ Visceral sensation $(0.4 \mathrm{ml})$} & $R$ & 0.916 & 0.848 & 0.788 \\
\hline & $\mathrm{P}$ value & 0.001 & 0.004 & 0.012 \\
\hline \multirow[t]{2}{*}{ Visceral sensation $(0.8 \mathrm{ml})$} & $R$ & 0.755 & 0.856 & 0.823 \\
\hline & $P$ value & 0.019 & 0.003 & 0.006 \\
\hline \multirow[t]{2}{*}{ Visceral sensation $(1.2 \mathrm{ml})$} & $R$ & 0.857 & 0.716 & 0.723 \\
\hline & $P$ value & 0.003 & 0.030 & 0.028 \\
\hline
\end{tabular}

Association between c-fos in CNS (frontal lobe, hippocampus, and cornu dorsale) and clinical parameters (general state, colonic motility, visceral sensation) was analyzed using Pearson's correlation test in IBS model rats $(n=30)$. c-fos in CNS significantly correlated with the clinical parameters $(P<0.05)$

$r$ Pearson correlation coefficient 
Patients enrolled in this study had no known intestinal infectious episode that could have triggered their IBS. HCs were recruited in outpatient department. Exclusion criteria included GI or hepatobiliary diseases, major abdominal surgery, allergic diseases, and other organic or severe psychiatric disorders as assessed by history taking, appropriate consultations, and laboratory tests. The use of nonsteroidal anti-inflammatory drugs, corticosteroids, and antimicrobial or immunosuppressive agents was not allowed.

All participants underwent colonoscopy after cleansing of the colon with magnesium sulfate performed in the evening before and the morning of the procedure. Three mucosal biopsy samples, taken from the rectosigmoid colon, were used for TH and c-fos assays. Briefly, three colonic biopsy samples of each study participant were rapidly immersed in diethyl pyrocarbonate normal saline and then transferred to Ependorf tubes in liquid nitrogen. The specimens were stored at $-80^{\circ} \mathrm{C}$ until use.

\section{Analysis of TH gene expression by real-time RT-PCR}

Quantitative real-time reverse transcription polymerase chain reaction (RT-PCR) assay was used to determine the messenger ribonucleic acid (mRNA) expression of TH in a fluorescent temperature cycler (TransGen Technology) following the manufacturer's recommendations. The colon was washed with PBS and then lysed in $1 \mathrm{ml}$ Trizol reagent, and the RNA was extracted. Total RNA was incubated with random primers at $70^{\circ} \mathrm{C}$ for $5 \mathrm{~min}, 37^{\circ} \mathrm{C}$ for $60 \mathrm{~min}$, and $70^{\circ} \mathrm{C}$ for $15 \mathrm{~min}$ in $\mathrm{RT}$ reaction buffer to make complementary deoxyribonucleic acid. PCRs were carried out in 96-well optical reaction plates. The $30 \mu \mathrm{l}$ amplification mixture contained $6 \mu \mathrm{RT}$ reaction products plus $\mathrm{MgCl}_{2}$ at $5 \mathrm{mmol} / \mathrm{l}$, each primer at $0.4 \mu \mathrm{mol} / \mathrm{l}$, and $2 \mu \mathrm{l}$ SYBR Green I mix (ABI Molecular Biochemicals). After initial denaturation at $95^{\circ} \mathrm{C}$ for $15 \mathrm{~min}$, reactions were cycled 40 times as follows: denaturation at $95^{\circ} \mathrm{C}$ for $15 \mathrm{~s}$, annealing at $60^{\circ} \mathrm{C}$ for $60 \mathrm{~s}$, and extension at $95^{\circ} \mathrm{C}$ for $15 \mathrm{~s}$. The amount of PCR products formed in each cycle was evaluated on the basis of SYBR Green fluorescence. At the end of each run, melting curve profiles were produced (cooling the sample to $60^{\circ} \mathrm{C}$ and heating slowly to $95^{\circ} \mathrm{C}$, with continuous measurement of fluorescence) to confirm amplification of specific transcripts. Cycle-to-cycle fluorescence emission readings were monitored and quantified using the second derivative maximum method of the software package. This method determined the crossing points of individual samples by an algorithm that identified the first turning point of the fluorescence curve. This turning point corresponded to the first maximum of the second derivative curve and correlated inversely with the $\log$ of the initial template concentration. TH mRNA levels were normalized with respect to $\beta$-actin level in each sample.
Primer sequences

Primer sequences for TH PCR amplification were: forward primer (5'-ATGTTGGCTGACCGCACATT-3') and reverse primer (5'-CCGAATTCCACAGTGAAC CAGT-3'); the PCR product obtained was 108 bp long. $\beta$-actin ribosomal RNA was used as internal reference. Primer sequences were: forward primer $5^{\prime}$-CATTGCT GACAGGATGCAGAAG-3' and reverse primer 5'GAGCCACCAATCCACACAGAGT-3'; the PCR product obtained was 107 bp long.

Data and statistical analysis

All data are expressed as means \pm SE. Demographic and personal characteristics were summarized as mean values and standard deviations. Control group and subgroup differences were assessed with Student's $t$-tests. The Pearson's correlation test was used to analyze correlations between the c-fos activity in CNS (frontal lobe, hippocampus, and cornu dorsale) and the clinical parameters such as general state, colonic motility, and colonic sensation. All statistical analyses were executed using SPSS version 17.0 (SPSS, Chicago, IL, USA). Results that show statistical significance $(P<0.05)$ are reported. A $P$ value of $<0.05$ was considered statistically significant.

\section{Results}

c-fos highly expressed in CNS of IBS animal model

We analyzed expression of c-fos in CNS (frontal lobe, hippocampus, and cornu dorsale). c-fos in CNS of IBS animal model were significantly increased compared with control samples $(P=0.001, P=0.002$, and $P=0.002$, respectively). The c-fos in colon of IBS model was lightly higher than that of control; however, the difference did not reach significance ( $P=0.207$; Fig. 2$)$.

The correlation between expression of c-fos in CNS and clinical parameters of IBS animal model

Compared with control animals, colonic motility (fecal pellets) and visceral sensation (numbers of abdominal muscle contraction induced by $\mathrm{CRD}$ ) were significantly higher in IBS rats, while the general state (reduction of body weight gain) decreased significantly $(P<0.05)$. The results were consistent with the prior study [21]. In IBS model rats, the clinical parameters (general state, colonic motility, and visceral sensation) correlated with the c-fos in CNS (frontal lobe, hippocampus, and cornu dorsale) significantly $(P<0.05$; Table 1$)$. 
Fig. 1 No histopathological abnormalities could be seen in both animal model of IBS and controls. a The HE staining of colon of control rat $(\times 400)$. b The HE staining of colon of IBS model rat $(\times 400)$

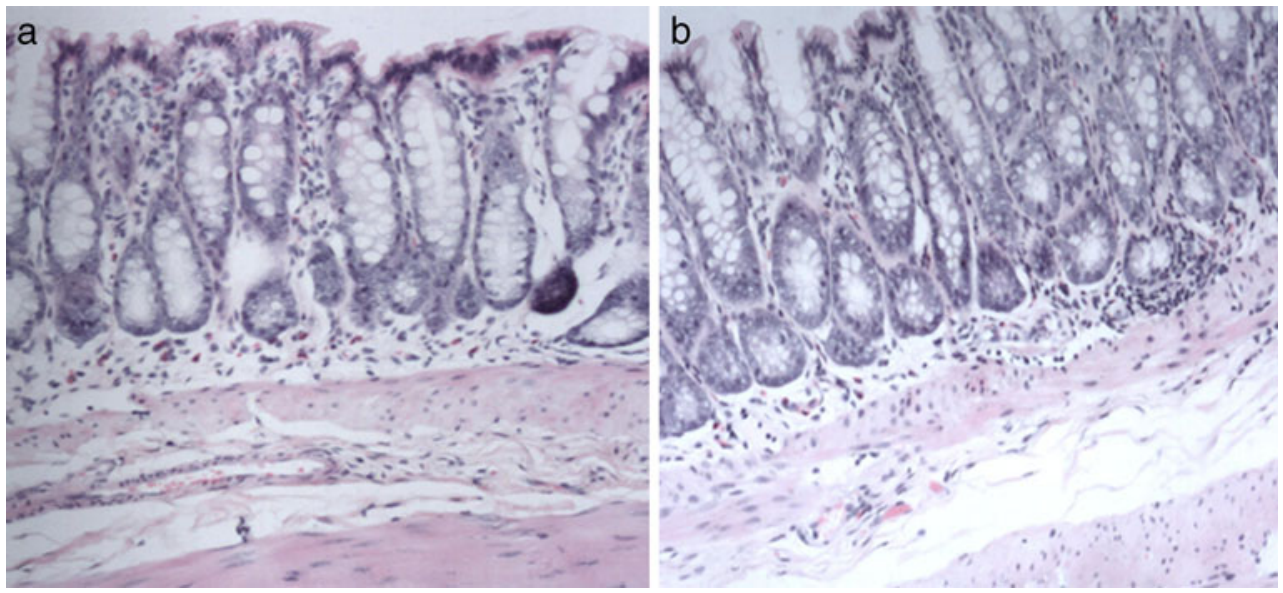

HE staining showed the pathologic histology of colon in animal model of IBS and control rats. It could be seen that the every layer tissue including the crypt, epithelium, and so on is complete, without histopathologic abnormalities in both groups (Fig. 1). However, compared with control rats, the enterochromaffin (EC) cell and serotonin (5-HT) in the colon of IBS model rats increased significantly $(P<0.05)$. Furthermore, as far as IBS model rats were concerned, c-fos in CNS showed a significant correlation with EC cell and 5HT in colon (Figs. 2, 3 and 4, Tables 2 and 3).
The possible mechanism of altered noradrenergic system in IBS

The anxiety and depression scores of IBS patients were significantly higher than those of controls

Clinical characteristics, such as age and sex ratio, were similar between IBS patients and controls $(P>0.05)$. However, IBS patients had significantly higher anxiety and depression scores than controls $(P<0.01$; Table 4$)$.
Fig. 2 The expression of c-fos in frontal lobe, hippocampus, and cornu dorsale of IBS model $(n=30)$ were significantly higher compared with controls $(n=28$; $P<0.05$ ); but c-fos in colon of IBS model and control were similar $(P>0.05)$. Middle line indicates medians, and the $u p$ and lower lines indicate interquartile (5th and 95th percentiles). a c-fos in frontal lobe of IBS model and control. b c-fos in hippocampus of IBS model and control. c c-fos in cornu dorsale of IBS model and control. $\mathbf{d ~ c}$-fos in colon of IBS model and control
A

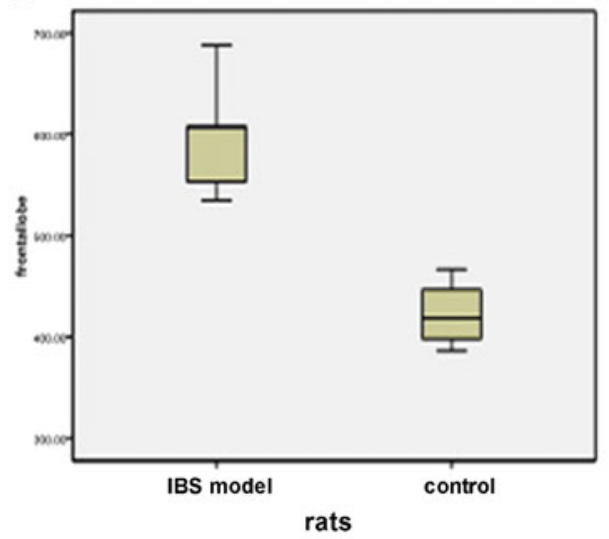

C

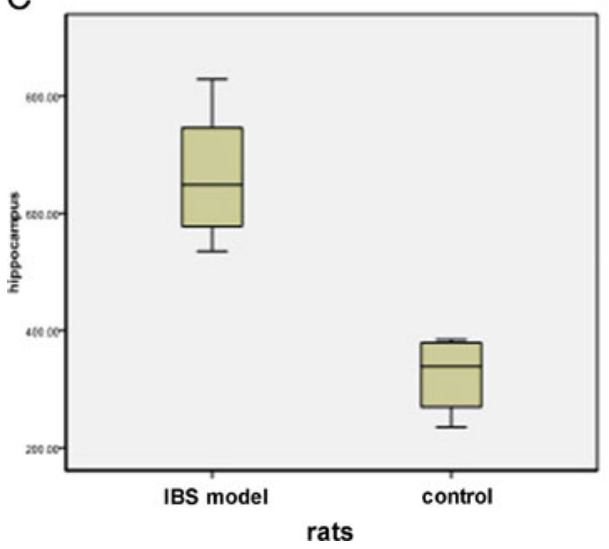

B

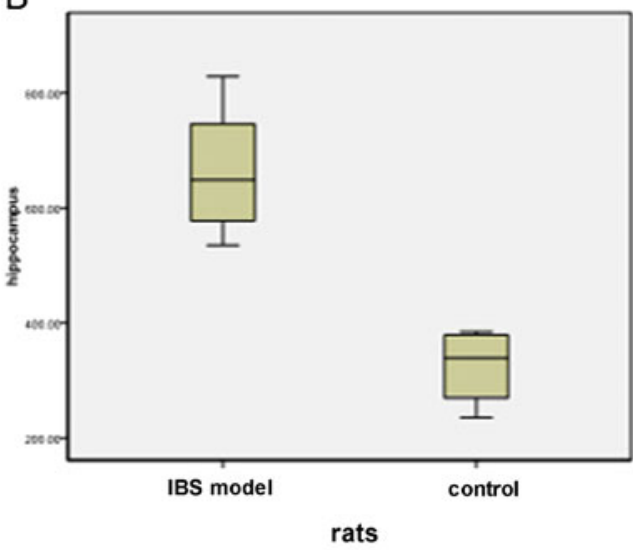

D

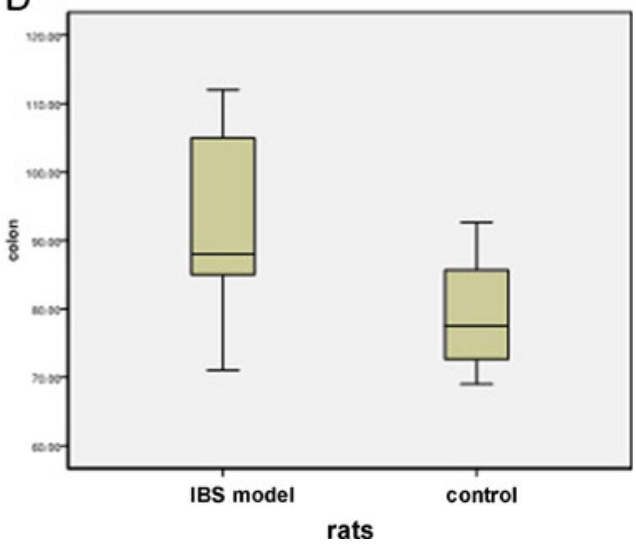


Fig. 3 The c-fos staining in the frontal lobe of IBS model and control. The c-fos staining can be seen both in cytoplasm and nucleus. The yellow-brown cfos positive neurons distribute irregularly in frontal lobe. The verge of cell is distinct. The morphology of cyton can be spindle-shaped, oval, or irregular. a c-fos in frontal lobe of IBS model $(\times 400)$. b c-fos in frontal lobe of control $(\times 400)$
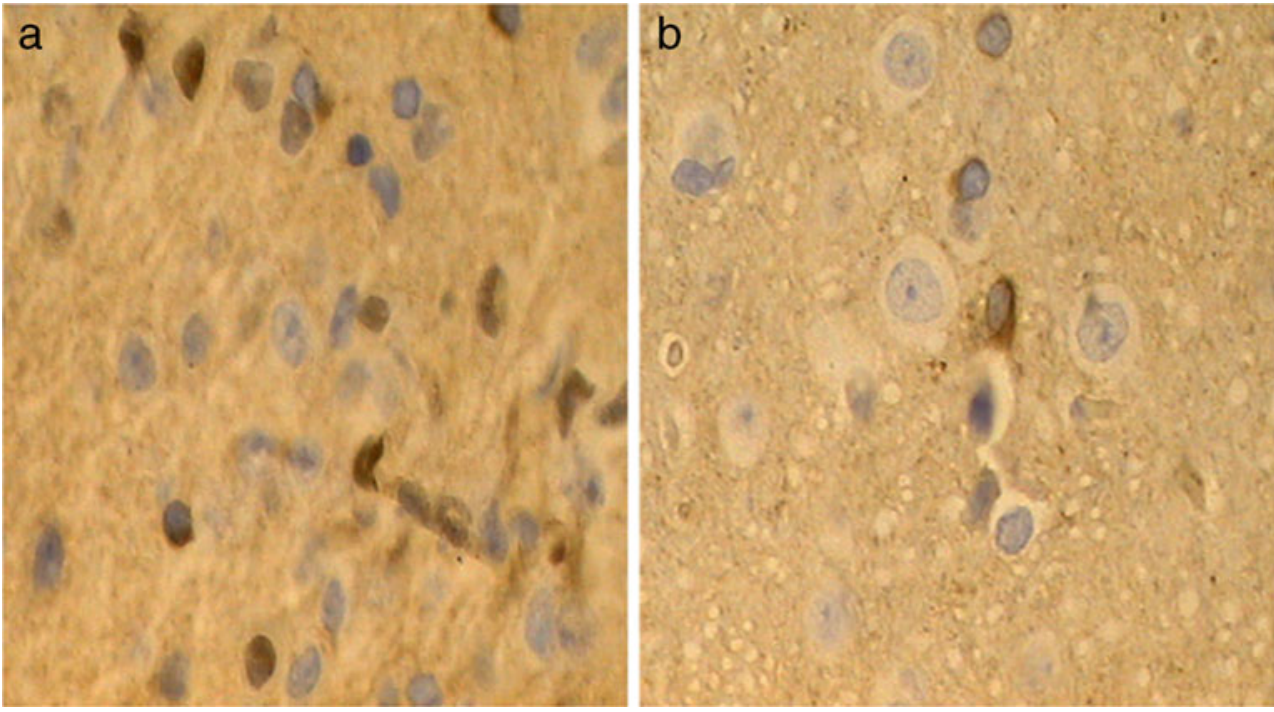

The expression of TH mRNA of IBS-D patients was almost four times as much as that of controls

The expression of TH mRNA in human colonic mucosa from IBS patients was slightly higher than that from HCs $(P>0.05)$. Furthermore, similar tendency could be seen between patients with IBS-D and IBS-C. Though there was no significant difference, the expression of TH mRNA of IBS-D patients was almost four times as much as that of HCs $(P>0.05$; Table 5$)$.

TH gene expression decreased in IBS patients with depressive disorder

Compared with IBS patients with lower standard deviation score (SDS), the $\mathrm{TH}$ gene expression decreased significantly in IBS patients with higher SDS $(P<0.05)$. But no significant difference of TH gene could be seen between IBS patients with higher and lower SAS $(P>0.05$; Table 6).

\section{Discussion}

The previous studies about significance of NE system in IBS focused on altered NE level in body fluid (plasma, urine, and saliva) [15-18]. At the same time, most studies whose investigative objects were tissues of IBS patients were done with attention to serotonin (5-HT) system in the colon of IBS patients $[25,26]$. Until now, the significance of NE system in colon and the c-fos expression in CNS in IBS have not been reported. Therefore, our study firstly

Fig. 4 The yellow-brown 5-HT staining can be seen in cytoplasm of rat's colonic cell. a 5HT in colon of IBS mo $(\times 400)$. b $5-\mathrm{HT}$ in colon of control $(\times 400)$
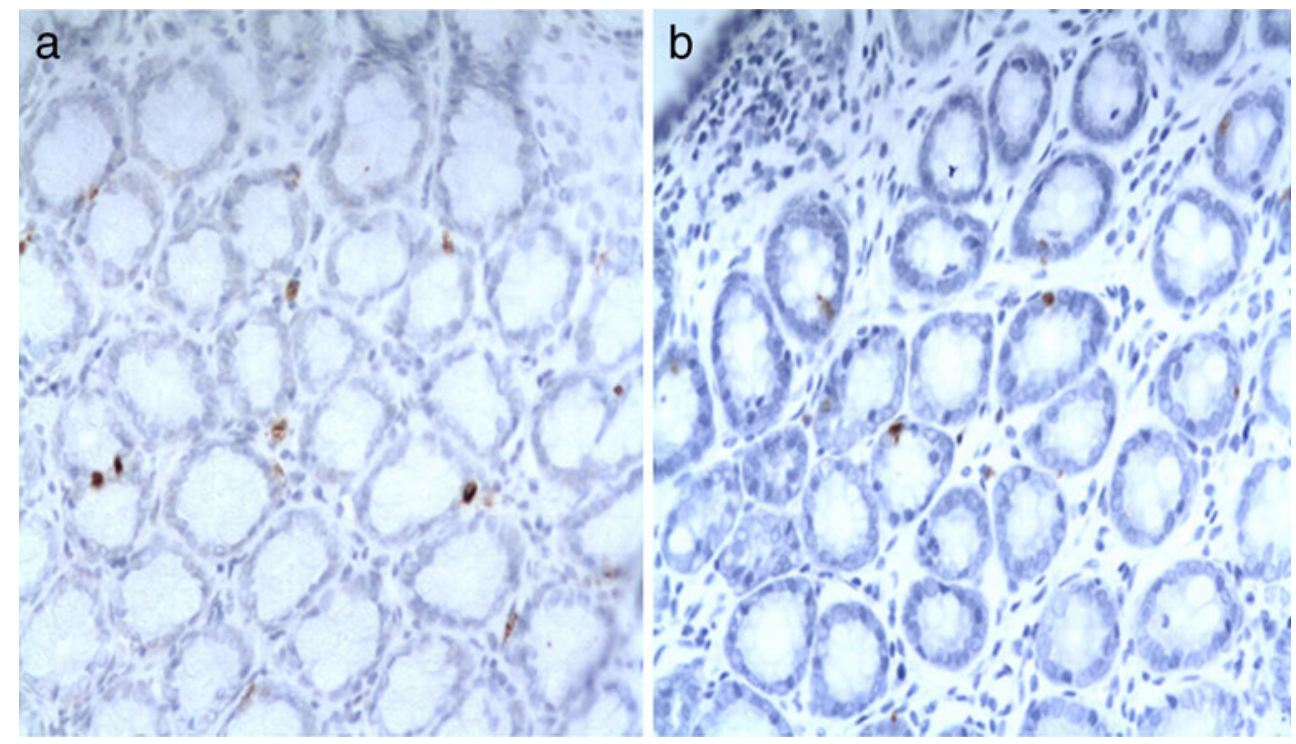
Table 2 The 5-HT and EC cells in the colon of rats

\begin{tabular}{lcc}
\hline & 5-HT in colon & EC cell in colon \\
\hline IBS model rats $(n=30)$ & $10.11 \pm 3.59^{*}$ & $22.367 \pm 22.37^{*}$ \\
Control $\operatorname{rats}(n=28)$ & $3.41 \pm 1.07$ & $12.17 \pm 2.52$ \\
\hline
\end{tabular}

Compared with control rats, the serotonin $(5-\mathrm{HT})$ and EC cell in the colon of IBS model rats increased significantly $(P<0.05)$. Values are shown as mean $\pm \mathrm{SD}$

discovered the following highlights. First, we used the brain-gut interaction animal model of IBS, which was proven well repeatable in the previous study [21], to investigate the activity of CNS and colon of IBS (assessed by expression of c-fos) and found that the animal model of IBS was associated with increased c-fos in CNS but normal c-fos in colon. Second, we found the elevated c-fos in CNS was associated with colonic motility and sensation of animal model of IBS. In addition, correlation analysis was used to investigate the relationship between increased c-fos in CNS and serotonin in colon of IBS model. Thirdly, we used the colonic tissue obtained from IBS patients and HCs to compare the NE signaling in the two groups. Furthermore, we tested the idea whether the NE signaling may play a role in the different manifestation of IBS-D and IBSC patients.

The therapeutic option of IBS, as a functional disease, need not involve an operation, so it is difficult to get tissue of CNS from patients with IBS. But recent human neuroimaging studies have emphasized the importance of altered CNS activity in the manifestation of IBS. So it is of great significance to get the tissue of CNS from animal model of IBS and to study the altered CNS activity. Our previous study established the brain-gut interaction animal model of IBS, and it could integrate major characteristics of IBS-D such as altered colonic motility, visceral hypersensitivity, and psychiatric disorder [21]. But the mechanism was unclear. We try to answer the question in the present study.

c-fos, a marker of activation of tissue cell, is a good object which can represent the activity of tissue. The
Table 4 Clinical characteristics of IBS patients and controls

\begin{tabular}{lcc}
\hline & IBS & Controls \\
\hline Male:female & $14: 15$ & $7: 8$ \\
Age & $41.5 \pm 13.6$ & $40.8 \pm 6.7$ \\
SAS & $43.1 \pm 8.9^{*}$ & $27.0 \pm 3.7$ \\
SDS & $49.6 \pm 10.0^{*}$ & $27.4 \pm 2.7$ \\
\hline
\end{tabular}

No difference could be seen between IBS patients and controls in age and sex ratio, but SAS and SDS of IBS patients were significantly higher $(P<0.01)$. Values are shown as mean $\pm \mathrm{SD}$

$S A S$ Self-rating anxiety scale, $S D S$ self-rating depression scale

present study showed that the animal model of IBS was associated with increased c-fos in CNS (frontal lobe, hippocampus, and cornu dorsale) but normal c-fos in colon, which showed that psychological stress might induce hyperexcitability of colon indirectly through activation of CNS. These data are consistent with the findings of the study of Macsharry et al. [27], which demonstrated an augmented colorectal distension-induced elevated c-fos in prefrontal cortex activity in Wistar rats similar to that seen in IBS patients. Taken together, c-fos expression in CNS is critical for induction of visceral hypersensitivity of IBS. In a word, the present study represented the first demonstration of significant molecular alterations specific to the CNS in IBS animal model and supported the assumption that psychological stress, such as the chronic and acute stress, might induce visceral hypersensitivity mainly via activation of CNS. That is, heightened CNS activity plays a very important role in pathophysiologic mechanism of IBS. These results provide a rational basis for current pharmacologic approaches involving regulation of CNS function in IBS patients.

Our data showed altered colonic motility and sensation were associated with the elevated c-fos in CNS seen in animal model of IBS. These data suggested that CNS may interact with colon and contribute to the altered colonic motility and sensation in IBS. And the strength of the frontal lobe to the phenotype maybe most. Thus

Table 3 Association between c-fos in CNS and serotonin in colon of IBS model rats

\begin{tabular}{llccr}
\hline & & c-fos in frontal lobe & c-fos in hippocampus & c-fos in cornu dorsale \\
\hline EC cell in colon & $R$ & 0.923 & 0.758 & 0.731 \\
\multirow{3}{*}{ 5-HT in colon } & $P$ value & 0.000 & 0.018 & 0.025 \\
& $R$ & 0.760 & 0.848 & 0.857 \\
& $P$ value & 0.017 & 0.004 & 0.003
\end{tabular}

In IBS model rats $(n=30)$, association between c-fos in CNS (frontal lobe, hippocampus, and cornu dorsale) and serotonin system (EC cells, 5HT) was analyzed using Pearson's correlation test. The results indicate that c-fos in CNS significantly correlates with serotonin system

$r$ Pearson correlation coefficient 
Table 5 The mRNA level of TH in human colonic mucosa

\begin{tabular}{lccc}
\hline & IBS-D patients $(n=19)$ & IBS-C patients $(n=10)$ & Controls $(n=15)$ \\
\hline TH mRNA & $0.10876 \pm 0.35896$ & $0.03470 \pm 0.05444$ & $0.02768 \pm 0.04521$ \\
\hline
\end{tabular}

The expression of TH mRNA of IBS-D patients was almost four times as much as that of HCs. Values are shown as mean \pm SD

pharmacogenomic studies focusing on the regulation of expression of c-fos would be of significant interest and potential clinical relevance in the treatment of IBS.

Correlation analysis was used to investigate the relationship between the expression of $\mathrm{c}$-fos in CNS and serotonin in the colon of model group. The results showed that the abnormal number of EC cells and 5-HT level were correlated with the increased c-fos in CNS, which supported the hypothesis that the activation of CNS may induce activation of EC cells in the colon and then the release of 5-HT. There is ample evidence for elevated level of 5-HT in the colon of IBS patients [2831]. EC cells are likely sources. This is also supported by the higher number of EC cells in IBS colonic tissue compared with controls and their correlation with 5-HT seen in the present study.

There is evidence for complex alterations in mediators released from the colonic mucosa [7, 8, 32-35]. Our prior study also found altered noradrenergic system in colon of IBS model [21]. In the present study, we focused on noradrenergic signaling $(\mathrm{TH})$ in colon of IBS patients for the first time, which is thought to play a major role in IBS pathophysiology. Although likely, it remains speculative how altered noradrenergic signaling contributes to dysfunctions in IBS patients such as alterations in colonic motility, sensation, or secretion. Intriguingly, the current results did not find difference in synthesis of NE (TH mRNA) between IBS patients and HC. How can we explain it? The specific NE reuptake transporter (NET) is required to terminate the action of $\mathrm{NE}$ and is very important for controlling the neural signal intensity. The role of $\alpha_{2 \mathrm{~A}}$-adrenoreceptor (AR) is presynaptic inhibition, so downregulation of NET and $\alpha_{2 \mathrm{~A}}$-AR protein will result in increased release of NE. Our prior study found that
NET and $\alpha_{2 \mathrm{~A}}$-AR in colon of IBS model decreased significantly compared with controls [21]. So we speculate that, instead of resulting from abnormality of $\mathrm{TH}$, the altered NE system in IBS is probably the result of other molecular mechanism as follows: (1) the increased c-fos in CNS, (2) the downregulation of NET and $\alpha_{2 \mathrm{~A}}$-AR protein in colon, or (3) though there was no significant difference, the expression of TH mRNA of IBS-D patients was almost four times as much as that of HCs. Thus the negative result about TH in our study was probably because of the minor sample size.

The present study shows, for the first time, that altered noradrenergic signaling in colonic mucosa might be a relevant factor in the pathophysiology of IBS, but the synthesis of NE may not be the key. Although IBS-D and IBS-C patients have distinct pathophysiologic properties, these data showed that the expression of TH in IBS-D and IBS-C patients was similar. Therefore, the data suggested that some shared defects in noradrenergic signaling might underlie the altered motility, secretion, and sensation in IBS-D and IBS-C patients.

Recent studies confirm that impairment of the noradrenergic system is believed to participate in the pathogenesis of depressive disorders [36-38]. The present study found a decrease in TH gene expression in colon of IBS patients with higher SDS scale. We speculated that the decrease in $\mathrm{TH}$ will result in hypofunction of noradrenergic system and the hypofunction will further induce depression-related symptoms. Taken together, the present study showed that the IBS patients with depression demonstrated specific change in gene expression of $\mathrm{TH}$, which indicates that drugs strengthening noradrenergic function maybe useful in the treatment of IBS patients with depressive disorder.

Table 6 TH gene expression in IBS patients with different mood disorder

\begin{tabular}{llll}
\hline IBS patients & TH mRNA & IBS patients & TH mRNA \\
\hline SDS $>50(n=12)$ & $0.01603 \pm 0.0095$ & SAS $>50(n=10)$ & $0.02173 \pm 0.01328$ \\
SDS $<50(n=17)$ & $0.1235 \pm 0.103$ & SAS $<50(n=19)$ & $0.1001 \pm 0.0839$ \\
$P$ & 0.042 & $P$ & 0.366 \\
\hline
\end{tabular}

Compared with IBS patients with lower SDS, the TH gene expression decreased significantly in IBS patients with higher SDS. But no significant difference of TH gene could be seen between IBS patients with higher and lower SAS 
Acknowledgement $\mathrm{Ru}$ Zhang is MD of Peking Union Medical College Hospital. After graduation, she works in Nanlou Department of Gastroenterology, Chinese PLA General Hospital now. The authors thank Dr. Xiaoou Yang for excellent secretarial assistance. Olympus $\mathrm{CH}-2$ microscope with the computer-assisted analysis system was the property of the Pathology Institute of Beijing Anzhen Hospital of the Capital University of Medical Science.

Funding This study was supported by grants from the National Natural Science Foundation of China (no. 30470795) and the Research Fund for the Doctoral Program of Higher Education of China (no. 20070023053). The authors have no conflicts of interest.

Open Access This article is distributed under the terms of the Creative Commons Attribution Noncommercial License which permits any noncommercial use, distribution, and reproduction in any medium, provided the original author(s) and source are credited.

\section{References}

1. Drossman DA, Camilleri M, Mayer EA et al (2002) AGA technical review on irritable bowel syndrome. Gastroenterology 123:2108-2131

2. Azpiroz F, Bouin M, Camilleri M et al (2007) Mechanisms of hypersensitivity in IBS and functional disorders. Neurogastroenterol Motil 19:62-88

3. Arebi N, Gurmany S, Bullas D et al (2008) Review article: the psychoneuroimmunology of irritable bowel syndrome - an exploration of interactions between psychological, neurological and immunological observations. Aliment Pharmacol Ther 28:830-840

4. Monnikes H, Tebbe JJ, Hildebrandt M et al (2001) Role of stress in functional gastrointestinal disorders. Evidence for stressinduced alterations in gastrointestinal motility and sensitivity. Dig Dis 19:201-211

5. Bearcroft CP, Perrett D, Farthing MJ (1998) Postprandial plasma 5-hydroxytryptamine in diarrhoea predominant irritable bowel syndrome: a pilot study. Gut 42:42-46

6. Camilleri M (2001) Management of the irritable bowel syndrome. Gastroenterology 120:652-668

7. Barbara G, Wang B, Stanghellini V et al (2007) Mast celldependent excitation of visceral-nociceptive sensory neurons in irritable bowel syndrome. Gastroenterology 132:26-37

8. Cenac N, Andrews CN, Holzhausen M et al (2007) Role for protease activity in visceral pain in irritable bowel syndrome. J Clin Invest 117:636-647

9. Buhner S, Li Q, Vignali S et al (2009) Activation of human enteric neurons by supernatants of colonic biopsy specimens from patients with irritable bowel syndrome. Gastroenterology 137:1425-1434

10. Brarucha AE, Camilleri M, Zinsmeister AR et al (1997) Adrenergic modulation of human colonic motor and sensory function. Am J Physiol 273:G997-G1006

11. Iovino P, Azpiroz F, Domingo E et al (1995) The sympathetic nervous system modulates perception and reflex responses to gut distention in humans. Gastroenterology 108:680-686

12. Carrasco GA, Van de Kar LD (2003) Neuroendocrine pharmacology of stress. Eur J Pharmacol 463:235-272

13. Patacchioli FR, Angelucci L, Dellerba G et al (2001) Actual stress, psychopathology and salivary cortisol levels in the irritable bowel syndrome (IBS). J Endocrinol Investig 24:173-177

14. Katayama M, Nomura K, Ujihara M et al (1998) Age-dependent decline in cortisol levels and clinical manifestations in patients with ACTH-independent Cushing's syndrome. Clin Endocrinol 49:311-316

15. Heitkemper M, Jarrett M, Levy R et al (2004) Self-management for women with irritable bowel syndrome. Clin Gastroenterol Hepatol 2:585-596

16. Elsenbruch S, Holtmann G, Oezcan D et al (2004) Are there alterations of neuroendocrine and cellular immune responses to nutrients in women with irritable bowel syndrome? Am J Gastroenterol 99:703-710

17. Posserud I, Agerforz P, Ekman R et al (2004) Altered visceral perceptual and neuroendocrine response in patients with irritable bowel syndrome during mental stress. Gut 53:1102-1108

18. Levine BS, Jarrett M, Cain KC et al (1997) Psychophysiological response to a laboratory challenge in women with and without diagnosed irritable bowel syndrome. Res Nurs Health 20:431-441

19. Galligan JJ (2004) 5-Hydroxytryptamine, ulcerative colitis, and irritable bowel syndrome: molecular connections. Gastroenterology 126:1897-1899

20. Kim HJ, Camilleri M, Carlson PJ et al (2004) Association of distinct alpha(2) adrenoceptor and serotonin transporter polymorphisms with constipation and somatic symptoms in functional gastrointestinal disorders. Gut 53:829-837

21. Zou N, Lv H, Li J et al (2008) Changes in brain G-proteins and colonic sympathetic neural signaling in chronic-acute combined stress rat model of IBS. Transl Res 152:283-289

22. Monnikes H, Lauer G, Arnold R (1997) Peripheral administration of cholecystokinin activates c-fos expression in the locus coeruleus/subcoeruleus nucleus, dorsal vagal complex and paraventricular nucleus via capsaicin-sensitive vagal afferents and CCK-A receptors. Brain Res 770:277-288

23. Traub RJ, Silva E, Gebhart GF et al (1996) Noxious colorectal distention induced-c-Fos protein in limbic brain structures in the rat. Neurosci Lett 215:165-168

24. Barone FC, Deegan JF, Price WJ et al (1990) Cold-restraint stress increases rat fecal pellet output and colonic transit. Am J Physiol 258:G329-G337

25. Atkinson W, Lockhart S, Whorwell PJ et al (2006) Altered 5hydroxytryptamine signaling in patients with constipation- and diarrhea-predominant irritable bowel syndrome. Gastroenterology 130:34-43

26. Coates MD, Mahoney CR, Linden DR et al (2004) Molecular defects in mucosal serotonin content and decreased serotonin reuptake transporter in ulcerative colitis and irritable bowel syndrome. Gastroenterology 126:1657-1664

27. Macsharry J, O’Mahony L, Fanning A et al (2008) Mucosal cytokine imbalance in irritable bowel syndrome. Scand J Gastroenterol 43:1467-1476

28. Kraneveld AD, Rijnierse A, Nijkamp FP et al (2008) Neuro-immune interactions in inflammatory bowel disease and irritable bowel syndrome: future therapeutic targets. Eur J Pharmacol 585:361-374

29. Aerssens J, Camilleri M, Talloen W et al (2008) Alterations in mucosal immunity identified in the colon of patients with irritable bowel syndrome. Clin Gastroenterol Hepatol 6:194-205

30. Liebregts T, Adam B, Bredack C et al (2007) Immune activation in patients with irritable bowel syndrome. Gastroenterology 132:913-920

31. Gibney SM, Gosselin RD, Dinan TG et al (2010) Colorectal distension-induced prefrontal cortex activation in the WistarKyoto rat: implications for irritable bowel syndrome. Neuroscience 165:675-683

32. Barbara G, Stanghellini V, de Giorgio R et al (2004) Activated mast cells in proximity to colonic nerves correlate with abdominal pain in irritable bowel syndrome. Gastroenterology 126:693-702

33. Guilarte M, Santos J, de Torres I et al (2007) Diarrhoeapredominant IBS patients show mast cell activation and hyperplasia in the jejunum. Gut 56:203-209 
34. Dunlop SP, Coleman NS, Blackshaw E et al (2005) Abnormalities of 5-hydroxytryptamine metabolism in irritable bowel syndrome. Clin Gastroenterol Hepatol 3:349-357

35. Kerckhoffs AP, Ter Linde JJ, Akkermans LM et al (2008) Trypsinogen IV, serotonin transporter transcript levels, and serotonin content are increased in small intestine of irritable bowel syndrome patients. Neurogastroenterol Motil 20:900 907

36. Orsetti M, Di Brisco F, Canonico PL et al (2008) Gene regulation in the frontal cortex of rats exposed to the chronic mild stress paradigm, an animal model of human depression. Eur J Neurosci 27:2156-2164

37. Dagnino-Subiabre A, Orellana JA, Carmona-Fontaine $\mathrm{C}$ et al (2006) Chronic stress decreases the expression of sympathetic markers in the pineal gland and increases plasma melatonin concentration in rats. J Neurochem 97:1279-1287

38. Duncko R, Kiss A, Skultétyová I et al (2001) Corticotropin-releasing hormone mRNA levels in response to chronic mild stress rise in male but not in female rats while tyrosine hydroxylase mRNA levels decrease in both sexes. Psychoneuroendocrinology 26:77-89 